\title{
LEVEL LOWERING MODULO PRIME POWERS AND TWISTED FERMAT EQUATIONS
}

\author{
SANDER R. DAHMEN AND SOROOSH YAZDANI
}

\begin{abstract}
We discuss a clean level lowering theorem modulo prime powers for weight 2 cusp forms. Furthermore, we illustrate how this can be used to completely solve certain twisted Fermat equations $a x^{n}+b y^{n}+c z^{n}=0$.
\end{abstract}

\section{INTRODUCTION}

Since the epoch making proof of Fermat's Last Theorem [15, 14, many Diophantine problems have been resolved using the deep methods developed for FLT and extensions thereof. One of the basic tools involved are so-called level lowering results, see e.g. [8, 9]. These provide congruences between modular forms of different levels. Until now, all applications of the modular machinery to Diophantine equations only involved level lowering modulo primes. Although recently a level lowering result modulo prime powers has been established [4, the statements there are not very fit for applications to Diophantine equations. The purposes of this paper are twofold. First of all, we give a clean level lowering result modulo prime powers that is suitable for applications to Diophantine equations. Second, we illustrate how this result can be applied, by completely solving certain twisted Fermat equations, i.e. Diophantine equations of the form

$$
a x^{n}+b y^{n}+c z^{n}=0 \quad x, y, z, n \in \mathbb{Z}, x y z \neq 0, n>1
$$

where $a, b, c$ are nonzero integers. For the twisted Fermat equations we consider, the genus one curve defined by $a x^{3}+b y^{3}+c z^{3}=0$ has infinitely many rational points, the curve defined by $a x^{9}+b y^{9}+c z^{9}=0$ has points everywhere locally, and level lowering modulo 3 also does not give enough information to deal with the exponent $n=9$ case. The main application of our level lowering modulo prime powers theorem is then to use level lowering modulo 9 to deal with the exponent $n=9$ case.

The organization of this paper is as follows. In Section 2 the level lowering result, Theorem 2, is stated and proved. In Section 3 we mainly deal with some issues related to irreducibility of mod 3 representations. In Section 4 we solve some twisted Fermat equations using level lowering modulo primes and level lowering modulo 9. Finally, in Section 5 we quickly discuss other possible methods to attack the twisted Fermat equation for exponent $n=9$, and we prove that standard level lowering modulo 3 methods can never work for our examples.

Date: August 31, 2010.

2010 Mathematics Subject Classification. Primary 11D41, 11F33; Secondary 11F11, 11F80, $11 \mathrm{G} 05$.

Key words and phrases. Modular forms, level lowering, Diophantine equations. 


\section{LEVEL LOWERING MODULO PRIME POWERS}

Let $N$ be a positive integer and $S_{2}\left(\Gamma_{0}(N)\right)$ denote the space of cuspidal modular forms of weight 2 with respect to $\Gamma_{0}(N)$. For any Hecke eigenform $f \in S_{2}\left(\Gamma_{0}(N)\right)$, denote by $K_{f}$ the field of definition of the Fourier coefficients of $f$, and by $\mathcal{O}_{f}$ its ring of integers. Note that the image of $f$ under different embeddings of $K_{f} \rightarrow \mathbb{C}$ gives conjugate Hecke eigenforms in $S_{2}\left(\Gamma_{0}(N)\right)$. As such, treating $K_{f}$ as an abstract number field and $f$ a modular form with Fourier coefficients in $K_{f}$ is akin to looking at $f$ and all its Galois conjugates at the same time. We say $f$ is a newform class of level $N$ if $f \in K[[q]]$ for a number field $K$, and the image of $f$ under each (equiv. under any) embedding of $K \rightarrow \mathbb{C}$ is a normalized Hecke newform in $S_{2}\left(\Gamma_{0}(N)\right)$. The degree of the newform class $f$ is the degree of the number field $K_{f}$. Denote by $G_{\mathbb{Q}}$ the absolute Galois group of $\mathbb{Q}$. Let $f$ be a newform class of level $N$. Given a prime $\lambda \subset \mathcal{O}_{f}$ lying above $l$, we can construct (see for example [13]) a Galois representation

for which

$$
\rho_{\lambda^{r}}^{f}: G_{\mathbb{Q}} \rightarrow \mathrm{GL}_{2}\left(\mathcal{O}_{f} / \lambda^{r}\right)
$$

- $\rho_{\lambda^{r}}^{f}$ is unramified away from $N l$,

- $\operatorname{trace}\left(\rho_{\lambda^{r}}^{f}\left(\operatorname{Frob}_{p}\right)\right) \equiv a_{p}(f)\left(\bmod \lambda^{r}\right)$ and $\operatorname{Norm}\left(\rho_{\lambda^{r}}^{f}\left(\operatorname{Frob}_{p}\right)\right) \equiv p\left(\bmod \lambda^{r}\right)$ for all primes $p \nmid N l$.

We remark that when $\rho_{\lambda}^{f}$ is absolutely irreducible, then $\rho_{\lambda^{r}}^{f}$ is uniquely determined (up to change of basis) for all positive integers $r$ by the congruences above.

Let $E / \mathbb{Q}$ be an elliptic curve of conductor $N$ and minimal discriminant $\Delta$. Let

$$
\rho_{l^{r}}^{E}: G_{\mathbb{Q}} \rightarrow \mathrm{GL}_{2}\left(\mathbb{Z} / l^{r} \mathbb{Z}\right)
$$

be the Galois representation coming from the natural Galois action of $G_{\mathbb{Q}}$ on $E\left[l^{r}\right](\overline{\mathbb{Q}})$. Assume that $N=N_{0} M$ with $N_{0}, M \in \mathbb{Z}_{>0}$ and that there is an odd prime $l$ such that

- $N_{0}$ and $M$ are coprime,

- $M$ is square free,

- for all primes $p \mid M$ we have $l \mid v_{p}(\Delta)$,

- $E[l]$ is irreducible (i.e. $\rho_{l}^{E}$ is an irreducible Galois representation).

Then by Ribet's level lowering ([8, [9]) there is a newform class of level $N_{0}$ and prime $\lambda \subset \mathcal{O}_{f}$ lying above $l$ such that

$$
\rho_{l}^{E} \simeq \rho_{\lambda}^{f}
$$

as Galois representations, or equivalently that $a_{p}(E) \equiv a_{p}(f)(\bmod \lambda)$ for all primes $p \nmid N_{0} l$.

It is natural to ask what happens when $l^{r} \mid v_{p}(\Delta)$ for all $p \mid M$ (see [2]). The situation in this case is more complicated. We first need to assume that $E[l]$ is strongly irreducible to get around some technical issues with deformation theory.

Definition 1. We say a 2-dimensional Galois representation $\rho$ of $G_{\mathbb{Q}}$ is strongly irreducible, if $\left.\rho\right|_{G_{\mathbb{Q}\left(\sqrt{l^{*}}\right)}}$ is absolutely irreducible for $l^{*}=(-1)^{(l-1) / 2} l$.

As noted in [4], using results of [10, when $l \geq 5$ and $E$ is semistable at $l$, then $\rho_{l}^{E}$ is strongly irreducible if it is irreducible. We will deal with the case $l=3$ for elliptic curves with full rational 2-torsion in Section 3 . 
We also need to assume that there is a unique newform class $f$ and an unramified prime ideal $\lambda$ at level $N_{0}$ to get the desired level lowering results.

Theorem 2. Let $E / \mathbb{Q}, N_{0}, M, l$ be as above. Assume that

- there is a positive integer $r$ such that for all primes $p \mid M$ we have $l^{r} \mid v_{p}(\Delta)$,

- for all primes $p \mid N_{0}$ we have $l \nmid v_{p}(\Delta)$,

- $l^{2} \nmid N$,

- $E[l]$ is strongly irreducible,

- there is a unique pair $(f, \lambda)$ with $f$ a newform class of level $N_{0}$ and $\lambda \subset \mathcal{O}_{f}$ an unramified prime lying above l such that $\rho_{l}^{E} \simeq \rho_{\lambda}^{f}$.

Then $\rho_{l^{r}}^{E} \simeq \rho_{\lambda^{r}}^{f}$. In particular, if all of the above assumptions are satisfied, then

(i) for all primes $p$ with $p \nmid l N$

$$
a_{p}(f) \equiv a_{p}(E) \quad\left(\bmod \lambda^{r}\right),
$$

(ii) for all primes $p$ with $p \nmid N_{0} l$ and $p \mid N$

$$
a_{p}(f) \equiv a_{p}(E)(1+p) \equiv \pm(1+p) \quad\left(\bmod \lambda^{r}\right) .
$$

Remark 3. Let us explain the reason for the assumptions made in this theorem. We need to assume $l^{2} \nmid N$, since the $R=\mathbb{T}$ results in this situation are not strong enough for our applications. The assumption that $\lambda$ is unramified is part of the uniqueness, in the sense that if $\lambda$ is ramified, then there are two Hecke eigenforms $f_{1}$ and $f_{2}$ in the same conjugacy class that are congruent to each other. Finally, we are assuming that $l \nmid v_{p}(\Delta)$ for $p \mid N_{0}$. We make this assumption since we want to guarantee that the Artin conductor of $\rho_{l}^{E}$ is $N_{0}$ and not something smaller. This way, we do not have to deal with oldforms for our analysis.

Remark 4. A similar theorem, using similar techniques, is proved in 4, although the statements of the main result there (specialized to our case) assume that $M$ is prime, $N$ is square free, and $l \nmid N$. Neither of these assumptions are necessary for the main proof, and in fact for applications to Diophantine equations these assumptions are usually not fulfilled.

We will present the proof of Theorem 2 for completeness. The proof uses standard Taylor-Wiles machinery ([15], [14, see also [3]) relating the deformation ring of modular Galois representations to a particular Hecke algebra. Specifically, let $f$ be a newform class of level $N_{0}$, and let $\lambda \subset \mathcal{O}_{f}$ be a prime lying above $l>2$. Recall that a lifting of $\rho_{\lambda}^{f}$ is a representation

$$
\rho: \mathrm{G}_{\mathbb{Q}} \longrightarrow \mathrm{GL}_{2}(R)
$$

where $R$ is a Noetherian complete local ring with the maximal ideal $\mathfrak{m}$ and the residue field $R / \mathfrak{m}=\mathcal{O}_{f} / \lambda$ such that $\rho \equiv \rho_{\lambda}^{f}(\bmod \mathfrak{m})$. A deformation of $\rho_{\lambda}^{f}$ is an equivalence class of such lifts. We say that $\rho$ is a minimal deformation of $\rho_{\lambda}^{f}$ if the ramification types of $\rho$ and $\rho_{\lambda}^{f}$ are the same at all primes $p$.

Assume that $\rho_{\lambda}^{f}$ is strongly irreducible and semistable at $l$. Then we know that there is a universal deformation ring $R^{\text {univ }}$ and a universal deformation $\rho^{\text {univ }}$ : $G_{\mathbb{Q}} \rightarrow \mathrm{GL}_{2}\left(R^{\text {univ }}\right)$ such that every minimal deformation $\rho_{\lambda}^{f}$ is strictly equivalent to a unique specialization of $\rho^{\text {univ }}$ under a unique homomorphism $R^{\text {univ }} \rightarrow R$. Let $\mathbb{T}$ be the Hecke algebra acting on $S_{2}\left(\Gamma_{0}\left(N_{0}\right)\right)$, completed at the maximal ideal 
corresponding to $\rho_{\lambda}^{f}$. If we assume that $N_{0}$ is the Artin conductor of $\rho_{\lambda}^{f}$, then we have a surjective map $\Phi: R^{\text {univ }} \rightarrow \mathbb{T}$. We have the following celebrated result.

Theorem 5 (Taylor-Wiles). Let $l$ be an odd prime. Assume that $\rho_{\lambda}^{f}$ is strongly irreducible. Then

$$
\Phi: R^{\text {univ }} \longrightarrow \mathbb{T}
$$

is an isomorphism and $R^{\mathrm{univ}}$ is a complete intersection.

Proof. For a proof when $\rho_{\lambda}^{f}$ is assumed to be semistable see [15, [14, or [1]. To prove the result stated here we also need Diamond's strengthening [3. We remark that in all of the above theorems, the statement proved is presented as $R_{Q}=\mathbb{T}_{Q}$ where $R_{Q}$ is a universal deformation ring for certain non-minimal deformations and $\mathbb{T}_{Q}$ is the completed Hecke algebra acting on $S_{2}\left(\Gamma_{Q}\right)$. The case that we are using is when $Q$ is the empty set. In this case $R_{\emptyset}=R$, however the group $\Gamma_{\emptyset}$ in the loc. cit. lies between $\Gamma_{0}(N)$ and $\Gamma_{1}(N)$. Fortunately this group is chosen in such a way that the space on which the diamond operator is acting trivially modulo $l$, is precisely $\Gamma_{0}(N)$. Therefore $\mathbb{T}_{\emptyset}=\mathbb{T}$.

As pointed out in $[4, R=\mathbb{T}$ results are the key to proving level lowering statements.

Proposition 6. Let $g$ be a newform class of level $N_{g}$ and degree 1 . Assume that there is a pair $(f, \lambda)$ with $f$ a newform class of level $N_{f}$ and $\lambda \subset \mathcal{O}_{f}$ an unramified prime lying above $l$, and a positive integer $r$ such that

- $\rho_{l}^{g}$ and $\rho_{l^{r}}^{g}$ have Artin conductor $N_{f}$,

- $\rho_{\lambda}^{f} \simeq \rho_{l}^{g}$,

- there is no other pair $\left(f^{\prime}, \lambda^{\prime}\right)$ with $f^{\prime}$ a newform class of level $N_{f}$ and $\lambda^{\prime} \subset \mathcal{O}_{f^{\prime}}$ a prime lying above l such that $\rho_{\lambda^{\prime}}^{f^{\prime}} \simeq \rho_{l}^{g}$,

- $\rho_{l}^{g}$ is strongly irreducible.

Then $\rho_{l^{r}}^{g} \simeq \rho_{\lambda^{r}}^{f}$.

Proof. Let $R^{\text {univ }}$ be the universal deformation ring for the minimal deformations of $\rho_{\lambda}^{f}$. By results of [14] we get that $R^{\text {univ }}=\mathbb{T}$. Since we are assuming that $g$ has rational integral coefficients and that $\rho_{\lambda}^{f} \simeq \rho_{l}^{g}$, we get that $\mathcal{O}_{f} / \lambda=\mathbb{Z} / l=\mathbb{F}_{l}$. Since we are also assuming that there is a unique $(f, \lambda)$, and that $\lambda$ is unramified, we get $\mathbb{T}=\mathcal{O}_{f, \lambda}=\mathbb{Z}_{l}$. Furthermore, we have that the Artin conductor of $\rho_{l^{r}}^{g}$ is $N_{f}$, therefore we get that $\rho_{l^{r}}^{g}$ is a minimal deformation of $\rho_{\lambda}^{f}$, hence it corresponds to a map $\mathbb{T} \rightarrow \mathbb{Z} / l^{r}$. However, there exists only one reduction map from $\mathbb{Z}_{l}$ to $\mathbb{Z} / l^{r}$, therefore $\rho_{l^{r}}^{g}$ is isomorphic to $\rho_{\lambda^{r}}^{f}$.

Remark 7. In Proposition [6 we are assuming that $g$ is of degree 1 to simplify the notation and the proof, and because this is the case we care most about in this paper. However, the proof does extend to the general case with some care.

We now give the proof of Theorem 2 ,

Proof. Let $E / \mathbb{Q}, N_{0}, M$, and $l$ be as required. In particular, assume that $E[l]$ is strongly irreducible. Since we are assuming that $l^{r} \mid v_{p}(\Delta)$ for all $p \mid M$ (and $l \nmid v_{p}(\Delta)$ for all $l \mid N_{0}$ ) we get that the Artin conductor of $\rho_{l^{r}}^{E}$ is $N_{0}$. By Ribet's level lowering we get that there is a newform class $f$ of level $N_{0}$ and a prime $\lambda$ such that $\rho_{\lambda}^{f} \simeq \rho_{l}^{E}$. Therefore, we can apply Proposition 6 to prove that $\rho_{l^{r}}^{E} \simeq \rho_{\lambda^{r}}^{f}$. As is well known, 
the congruences (i) and (ii) in the statement of the theorem follow by comparing the traces of Frobenius.

Remark 8. When $f$ is not unique, all hope is not lost and in favourable conditions, we can in fact get some explicit level lowering results. As an example, consider an elliptic curve $E / \mathbb{Q}$ of conductor $71 M$ and minimal discriminant $71 M^{27}$, for some square free positive integer $M$ coprime to 71 . Furthermore, assume that $E[3]$ is strongly irreducible. Such an elliptic curve certainly exists, for example when $M=2$ we have the elliptic curve $142 e 1$ in the Cremona database

$$
E: y^{2}+x y=x^{3}-x^{2}-2626 x+52244 \text {. }
$$

By Ribet's level lowering, we can find a newform class $f$ of level 71 and a prime $\lambda \subset \mathcal{O}_{f}$ lying above 3 such that $\rho_{3}^{E} \simeq \rho_{\lambda}^{f}$. There are two newform classes $f_{1}$ and $f_{2}$, each of degree 3, whose complex embeddings span all of $S_{2}\left(\Gamma_{0}(71)\right)$. For $i=1,2$, we can check that $3 \mathcal{O}_{f_{i}}=\lambda_{i, 1} \lambda_{i, 2}$, where $\lambda_{i, 1}$ is of inertia degree one, while $\lambda_{i, 2}$ is of inertia degree 2. The image of $\rho_{\lambda_{i, 2}}^{f_{i}}$ is not contained in $\mathrm{GL}_{2}\left(\mathbb{F}_{3}\right)$, therefore $\rho_{\lambda_{i, 2}}^{f_{i}} \not \rho_{3}^{E}$. By computing some Fourier coefficients, we get that $\rho_{\lambda_{1,1}}^{f_{1}} \simeq \rho_{\lambda_{2,1}}^{f_{2}}$. We conclude that $\rho_{l}^{E}$ is isomorphic to both of these representations. Therefore, all the conditions of our level lowering result are fulfilled, except for the uniqueness of $(f, \lambda)$. This prevents us from proving a level lowering result modulo 27. However, by studying the deformation ring explicitly, we can still prove a level lowering result modulo 9 in the following way. For $i=1,2$, we compute that $\mathcal{O}_{f_{i}}$ is generated by $a_{5}\left(f_{i}\right)$, explicitly

$$
\begin{aligned}
& \mathcal{O}_{f_{1}}=\mathbb{Z}[t] /\left\langle t^{3}-5 t^{2}-2 t+25\right\rangle, \\
& \mathcal{O}_{f_{2}}=\mathbb{Z}[t] /\left\langle t^{3}+3 t^{2}-2 t-7\right\rangle .
\end{aligned}
$$

Furthermore, the full Hecke algebra acting on $S_{2}\left(\Gamma_{0}(71)\right)$ has the representation

$$
\mathbb{Z}[t] /<\left(t^{3}-5 t^{2}-2 t+25\right)\left(t^{3}+3 t^{2}-2 t-7\right)>
$$

where $t=T_{5}$ is the fifth Hecke operator. Therefore, the universal deformation ring of $\rho_{3}^{E}$, which is the localization of the Hecke algebra at $\lambda_{i, 1}$, is

$$
\left.\mathbb{T}=\mathbb{Z}_{3}[t] /<\left(t-\alpha_{1}\right)\left(t-\alpha_{2}\right)\right\rangle,
$$

where $\alpha_{1} \equiv 20(\bmod 27)$ and $\alpha_{2} \equiv 11(\bmod 27)$. Notice that $\alpha_{1} \equiv \alpha_{2} \equiv 2(\bmod 9)$, which means $\rho_{\lambda_{1,1}^{2}}^{f_{1}} \simeq \rho_{\lambda_{2,1}^{2}}^{f_{2}}$, a result that can also be read off from the Fourier coefficients of $f_{1}$ and $f_{2}$. Since $\rho_{27}^{E}$ is unramified away from 3 and 71 , and it is flat at 3 , we have that $\rho_{27}^{E}$ is a minimal deformation of $\rho_{3}^{E}$, hence it corresponds to a unique map $R^{\text {univ }} \rightarrow \mathbb{Z} / 27 \mathbb{Z}$. Note that this gives us two possible maps

$$
\begin{aligned}
\psi_{i}: & \mathbb{T} \rightarrow \mathbb{Z}_{3} \\
& t \mapsto \alpha_{i},
\end{aligned}
$$

corresponding to the two modular forms with coefficients in $\mathbb{Z}_{3}$. Let $\psi: \mathbb{T} \rightarrow \mathbb{Z} / 27 \mathbb{Z}$ correspond to $\rho_{27}^{E}$. Note that $\psi$ is uniquely defined by the image of $t$, and there are three possible choices for this image: 2,11 , or 20 . Reducing $\psi$ modulo 9 we get $\bar{\psi}: \mathbb{T} \rightarrow \mathbb{Z} / 9 \mathbb{Z}$ is given by $\bar{\psi}(t)=2$. Furthermore, $\bar{\psi}$ corresponds to $\rho_{9}^{E}$, so we get 
the following commutative diagram.

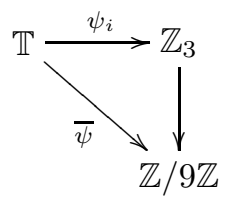

By universality, the map

$$
\mathbb{T} \stackrel{\psi_{i}}{\longrightarrow} \mathbb{Z}_{3} \longrightarrow \mathbb{Z} / 9 \mathbb{Z}
$$

corresponds to the reduction of the $\lambda_{i}$-adic representation of $f_{i}$ modulo $\lambda_{i}^{2}$, that is $\rho_{\lambda_{i}^{2}}^{f_{i}}$. Since this is the same as $\bar{\psi}$, which corresponds to $\rho_{9}^{E}$, we get that $\rho_{9}^{E} \simeq \rho_{\lambda_{i}^{2}}^{f_{i}}$ for $i=1$ and 2 .

In case we take for $E$ the elliptic curve $142 e 1$, we can check explicitly that $\rho_{27}^{E} \nsucceq \rho_{\lambda_{i, 1}^{3}}^{f_{i}}$ for $i=1$ or 2 , since $a_{5}(E)=2$, while $a_{5}\left(f_{i}\right) \equiv \alpha_{i} \not \equiv 2\left(\bmod \lambda_{i, 1}^{3}\right)$. The congruence modulo 9 can be verified by computing some Fourier coefficients explicitly.

\section{IRreducibility MOD 3}

In this section, we obtain a criterion for proving that $\rho_{3}^{E}$ is strongly irreducible when $E / \mathbb{Q}$ has a full rational 2-torsion structure. We start with a simple lemma.

Lemma 9. Let $E / \mathbb{Q}$ be an elliptic curve. If $\rho_{3}^{E}$ is irreducible, but not strongly irreducible, then $\rho_{3}^{E}\left(G_{\mathbb{Q}}\right)$ is contained in the normalizer of a split Cartan subgroup of $\mathrm{GL}_{2}\left(\mathbb{F}_{3}\right)$.

Proof. A (short) proof can be found in [11, Proposition 6].

Next, a lemma which restricts the possibility of the image of a mod-3 Galois representation attached to an elliptic curve over $\mathbb{Q}$ with full rational 2-torsion.

Lemma 10. Let $E / \mathbb{Q}$ be an elliptic curve with full rational 2 -torsion. Then $\rho_{3}^{E}\left(G_{\mathbb{Q}}\right)$ is not contained in the normalizer of a split Cartan subgroup of $\mathrm{GL}_{2}\left(\mathbb{F}_{3}\right)$.

Proof. Consider the modular curves $X_{\text {split }}(3), X(2), X(1)$ and denote by $j_{2}$ and $j_{\text {split }, 3}$ the $j$-maps from $X(2)$ to $X(1)$ and $X_{\text {split }}(3)$ to $X(1)$ respectively. We have explicitly

and

$$
j_{2}(s)=2^{8} \frac{\left(s^{2}+s+1\right)^{3}}{(s(s+1))^{2}}
$$

$$
j_{\text {split }, 3}(t)=12^{3}\left(\frac{4 t+4}{t^{2}-4}\right)^{3} .
$$

This allows us to explicitly compute the fiber product $X_{\text {split }}(3) \times_{X(1)} X(2)$ by equating $j_{2}(s)=j_{\text {split, } 3}(t)$, and we let $X$ to be the desingularization of this fiber product. We compute that $X$ has genus 1 and 6 cusps, all contained in $X(\mathbb{Q})$. We turn $X$ into an elliptic curve over $\mathbb{Q}$ by taking one of the cusps as the origin. Now $X$ is isomorphic over $\mathbb{Q}$ to the elliptic curve determined by

$$
y^{2}=x^{3}-15 x+22 \text {. }
$$

This curve has rank 0 and torsion group of order 6 . This shows that $X(\mathbb{Q})$ is exactly the set of cusps, which proves the proposition. 
Corollary 11. Let $E / \mathbb{Q}$ be an elliptic curve with full rational 2-torsion. If $\rho_{3}^{E}$ is irreducible, then $\rho_{3}^{E}$ is strongly irreducible.

Proof. This follows immediately by combining Lemma 9 and Lemma 10 ,

We still need to a nice criterion for deciding that $\rho_{3}^{E}$ is irreducible.

Lemma 12. Let $\mathcal{E} / \mathbb{F}_{p}$ be an elliptic curve over $\mathbb{F}_{p}$ and let a be the trace of Frobenius of this curve. Then $\mathcal{E}$ has a 3 -isogeny if and only if $a \equiv \pm(p+1)(\bmod 3)$.

Proof. Note that $\mathcal{E}$ has a 3 -isogeny, if and only if either $\mathcal{E}$ or its quadratic twist $\mathcal{E}^{\prime}$ has a 3 -torsion point, i.e. $3 \mid \# \mathcal{E}\left(\mathbb{F}_{p}\right)=p+1-a$ or $3 \mid \# \mathcal{E}^{\prime}\left(\mathbb{F}_{p}\right)=p+1+a$. This proves the lemma.

This brings us to the criterion we need for checking strong irreducibility.

Proposition 13. Let $E / \mathbb{Q}$ be an elliptic curve with full rational 2-torsion and $p \equiv 1(\bmod 3)$ a prime of good reduction for $E$. If $3 \mid a_{p}(E)$, then $\rho_{3}^{E}$ is strongly irreducible.

Proof. Corollary 11 tells us that irreducibility and strong irreducibility in our situation are equivalent. If $\rho_{3}^{E}$ is reducible, then $E / \mathbb{Q}$ has a rational 3-isogeny, which implies $\bar{E} / \mathbb{F}_{p}$ has a rational 3-isogeny for all primes of good reduction $p$. By Lemma 12 this implies that if $p \equiv 1(\bmod 3)$, then we have $a_{p}(E) \neq 0(\bmod 3)$, which is the desired result.

\section{Twisted Fermat equations}

Let $a, b, c$ be pairwise coprime nonzero integers and $n>1$ an odd integer (the case $n$ even is trivial, due to our sign choices). We are interested in solving the Diophantine equation

$$
a x^{n}+b y^{n}+c z^{n}=0 .
$$

For $n>3$, we know that this equation defines a curve $C_{n}$ of genus greater than one, so by Faltings' theorem we get that $C_{n}(\mathbb{Q})$ is finite for any such $n$. In fact, in all the cases we will consider in this paper, we prove that $C_{n}(\mathbb{Q})$ is empty for all $n>3$, except for one trivial solution when $n=7$ in one of our examples. For $n>3$ a prime, we will use the modular methods following [6] and [5]. For $n=3$ however, $C_{3}(\mathbb{Q}) \neq \emptyset$ and the Jacobian of the curve $C_{3}$, given by the equation

$$
Y^{2}=X^{3}-2^{4} 3^{3}(a b c)^{2},
$$

is elliptic curve of positive rank in all the cases we are considering. The only remaining case is when $n=9$, and we note that for our examples $C_{9}$ has local points everywhere. We use our level lowering results modulo prime powers to show that $C_{9}(\mathbb{Q})$ is also empty.

From a Diophantine point of view, the main result is the following.

Theorem 14. Let $(a, b, c)$ be one of $\left(5^{2}, 2^{4}, 23^{4}\right),\left(5^{8}, 2^{4}, 37\right),\left(5^{7}, 2^{4}, 59^{7}\right),\left(7,2^{4}, 47^{7}\right)$, $\left(11,2^{4}, 5^{2} \cdot 17^{2}\right)$. Then for $n \in \mathbb{Z}_{\geq 2}$ with $n \neq 3$ the twisted Fermat equation (11) has no solutions $x, y, z$ in integers with $x y z \neq 0$.

Remark 15. Since we are choosing $a, b$, and $c$ all positive, proving that there are no solutions when $n$ is even is trivial. Of course, by different choice of signs, one has to work a little bit harder, and we leave those cases to the interested reader. 
In a sense, we have a complete description of the solutions for all the exponents $n>1$, since we can find explicit generators for the Mordell-Weil group of the elliptic curve associated to $C_{3}$ over $\mathbb{Q}$.

4.1. The modular method. We review how to use elliptic curves, modular forms, and Galois representations to approach Diophantine equations of the form (1), mainly following 5 . We remark that we deviate from loc. cit. by allowing $n$ to be a prime power instead of just a prime. Fix nonzero coprime integers $a, b, c$. Assume there is a solution in integers $(x, y, z, n)$ to (1), with $x y z \neq 0$ and $n \geq 3$ odd. Without loss of generality we assume that $b y^{n}$ is even and that $a x^{n} \equiv-1$ $(\bmod 4)$. We also assume that $a x^{n}, b y^{n}$, and $c z^{n}$ are pairwise coprime. Consider the Frey elliptic curve

$$
Y^{2}=X\left(X-a x^{n}\right)\left(X+b y^{n}\right) .
$$

This model is minimal at all odd primes. Furthermore, if $b \equiv 0(\bmod 16)$, then we can find a global minimal model over $\mathbb{Z}$

$$
E_{n,(x, y)}: Y^{2}+X Y=X^{3}+\frac{b y^{n}-a x^{n}-1}{4} X^{2}-\frac{a b x^{n} y^{n}}{16} X .
$$

We often simply write $E_{n}$, or even $E$, when the indices are understood from the context. We assume that the condition on $b$ is satisfied, since these are the only cases that we will consider (for the general situation the reader can refer to [5]). The minimal discriminant and the conductor of $E_{n}$ are given by

$$
\begin{aligned}
& \Delta\left(E_{n}\right)=\frac{\left(a b c x^{n} y^{n} z^{n}\right)^{2}}{2^{8}}, \\
& N\left(E_{n}\right)=\prod_{\substack{p \mid a b c x y z \\
p \text { odd prime }}} p .
\end{aligned}
$$

Consider the Galois representation

$$
\rho_{n}^{E_{n}}: G_{\mathbb{Q}} \rightarrow \mathrm{GL}_{2}(\mathbb{Z} / n \mathbb{Z})
$$

Assume $n=l^{r}$ is a prime power with $l$ an odd prime and $r$ a positive integer, and that $\rho_{l}^{E_{n}}$ is strongly irreducible. By Ribet's level lowering [8, [9] and the work of Wiles and Taylor-Wiles [15, [14, we know that $\rho_{l}^{E_{n}}$ arises from a newform class $f$ of level

$$
N_{0}=\prod_{\substack{p \mid a b c \\ p>2}} p .
$$

This means that there exists a prime ideal $\lambda \subset \mathcal{O}_{f}$ lying above $l$, such that

$$
\rho_{l}^{E_{n}} \simeq \rho_{\lambda}^{f} .
$$

Remark 16. Note that, since we are assuming that $a, b$, and $c$ are pairwise coprime to each other, if $p^{n} \nmid a b c$ with $p$ prime, then for showing that (10) has no nontrivial solutions, we can assume without loss of generality that $a x^{n}, b y^{n}$, and $c z^{n}$ are pairwise coprime. This is the case for all of our examples when $n>7$. In general, if for some prime $p$, we have that $p$ divides $a x^{n}, b y^{n}$ and $c z^{n}$, then it is possible that $E_{n}$ has additive reduction at $p$, however for odd primes $p$, a quadratic twist of $E_{n}$ will have a semistable reduction at $p$. We will replace $E_{n}$ by its appropriate quadratic twist (if necessary) for the rest of this paper. All of our computations in this case will be the same, except that we might end up at a level dividing $N_{0}$, 
however if we use the level $N_{0}$, the argument still goes through. (The situation at the prime 2 is a bit more subtle, however we do not have to deal with it when $n \geq 5$ in our examples.) Note that if we use the smaller level, the argument can be easier. For instance, in the example

$$
5^{7} x^{n}+2^{4} y^{n}+59^{7} z^{n}=0
$$

when $n=7$, we only need to consider newform classes of level 1 (of which there are none). This gives us a considerably easier contradiction. However, for sake of uniformity, we actually deal with newform classes of level 295 to show that this equation has no nontrivial solutions.

For every equation in Theorem 14, the level $N_{0}$ we need to consider is given in Table 1 By comparing traces of Frobenius, we obtain the congruences (i) and (ii) with $r=1$. If $(f, \lambda)$ is the unique pair of newform class $f$ of level $N_{0}$ and prime $\lambda \subset \mathcal{O}_{f}$ lying above $l$ that satisfies (5), $\lambda$ is unramified, and for all primes $p \mid N_{0}$ we have $l \nmid v_{p}\left(\Delta\left(E_{n}\right)\right)$, then we can apply Theorem 2 to $\rho_{n}^{E_{n}}$. In this case we get that $\rho_{n}^{E_{n}} \simeq \rho_{\lambda^{r}}^{f}$ and in particular that the congruences (i) and (ii) hold.

4.1.1. $l \geq 5$. Let $l \geq 5$ be a prime number. Note that by the arguments of [12, Proposition 6] we have $\rho_{l}^{E_{l}}$ is irreducible, since $E$ is semistable and has full rational 2-torsion (by our earlier remarks, this tells us that $\rho_{l}^{E_{l}}$ is strongly irreducible). In order to prove that there are no solutions to (11) for $n=l$, it suffices to find a contradiction (using congruences) to (5) for all pairs $(f, \lambda)$ of newform classes $f$ of level $N_{0}$ and primes $\lambda \subset \mathcal{O}_{f}$ lying above $l$.

Let $f$ be a newform class of level $N_{0}$ such that (5) holds for some $\lambda$. For any prime $p$, define

$$
\mathcal{A}_{p}=\left\{\begin{array}{l}
\{a \in \mathbb{Z}: a \equiv p+1 \quad(\bmod 4) \text { and }|a| \leq 2 \sqrt{p}\} \quad \text { if } p \text { is odd } \\
\{-1,1\} \quad \text { if } p=2 .
\end{array}\right.
$$

We claim that for all primes $p$ where $E$ has good reduction we have $a_{p}(E) \in$ $\mathcal{A}_{p}$. This is because $E$ has full rational 2-torsion and for an odd prime $p$ of good reduction, $E[2]$ injects into the reduction of $E$ modulo $p$. If $E$ has good reduction at $p=2$, then one checks that the reduction of $E$ modulo $p$ still has a rational 2 -torsion point. Together with the Weil bound, the claim follows. Next, define for all primes $p$ the set

$$
\mathcal{T}_{p}=A_{p} \cup\{ \pm(p+1)\} .
$$

The congruences (i)](ii)] with $r=1$ now give us that for a prime $p \nmid N_{0}$ we have

$$
l \mid L_{f, p}=p \prod_{a \in \mathcal{T}_{p}} \operatorname{Norm}\left(a-a_{p}(f)\right) .
$$

(If the degree of $f$ is equal to 1 , then the prime $p$ before the product is not necessary, but in all our examples this does not lead to any new information.) It is of course possible that $L_{f, p}=0$, in which case $l \mid L_{f, p}$ does not give any information. However, all our examples are chosen such that, either $f$ is not rational, or it is rational and the elliptic curve of conductor $N_{0}$ associated to it by the Eichler-Shimura relation is not isogenous to an elliptic curve with full rational 2-torsion. In what follows, assume that $f$ satisfies these conditions. This implies that for infinitely many (in fact, a positive proportion of) primes $p$ we have $a_{p}(f) \notin \mathcal{A}_{p}$ (and hence $a_{p}(f) \notin \mathcal{T}_{p}$ since by the Weil bounds $a_{p}(f) \neq \pm(p+1)$ ). It is easy to get an upper bound in 
terms of $N_{0}$ (or $\left.a, b, c\right)$ for the smallest prime $p \nmid N_{0}$ for which $a_{p}(f) \notin \mathcal{A}_{p}$. In practice, for several primes $p_{\max }$, we compute

$$
\operatorname{gcd}\left\{L_{f, p}: \text { primes } p \leq p_{\max } \text { with } p \nmid N_{0}\right\}
$$

and the set of odd primes dividing this quantity, denoted $\mathcal{L}_{f, p_{\max }}$. We do this until we find a prime $p_{\max }$ for which $\mathcal{L}_{f, p_{\max }}$ is not empty and appears to be the same as $\mathcal{L}_{f, p_{\max }^{\prime}}$ for any prime $p_{\max }^{\prime} \geq p_{\max }$. This yields the information that for all primes $l>3$ such that $l \notin \mathcal{L}_{f, p_{\max }}$ we have a contradiction to (5) for all primes $\lambda \subset \mathcal{O}_{f}$ lying above $l$.

For the given level $N_{0}$, let us finally define for a prime $p_{\max }$ the set

$$
\mathcal{L}_{p_{\max }}=\bigcup_{f} \mathcal{L}_{f, p_{\max }}
$$

where the union is over all newform classes $f$ of level $N_{0}$. By taking $p_{\max }$ to be the maximum of the $p_{\max }$ 's for the newform classes $f$, we arrive at a finite set of odd primes $\mathcal{L}_{p_{\max }}$ which contains 3 and, in practice, just a few other odd primes. For every equation in Theorem 14, a value of $p_{\max }$ together with $\mathcal{L}_{p_{\max }}-\{3\}$ is given in Table 1. The significance for the original Diophantine problem is that for every odd prime $l \notin \mathcal{L}_{p_{\max }}$ we have that (1) has no integer solutions with $x y z \neq 0$.

In our examples, for the finitely many primes $l \geq 5$ contained in $\mathcal{L}_{p_{\max }}$, we show that $C_{l}(\mathbb{Q})=\emptyset$ (except for the one trivial exception) either by finding a prime $p$ for which $C_{n}\left(\mathbb{Q}_{p}\right)=\emptyset$ or, if no such prime $p$ exists, by using Kraus' method of reduction, see [7] or [5, Section 1.2.], which we briefly describe now.

Fix an exponent $n=l^{r}$ a prime power (in loc. cit. $n$ is assumed to be a prime). The possibilities for $a_{p}\left(E_{n}\right)\left(\right.$ and $\left.\operatorname{trace}\left(\rho_{l}^{E_{n}}\left(\operatorname{Frob}_{p}\right)\right)\right)$ with $p \equiv 1(\bmod l)$ sometimes can be shown to be strictly smaller than $\mathcal{A}_{p}$, (and $\mathcal{T}_{p}$ respectively) by using the additional information that (11) has to be satisfied modulo $p$. Let $p \nmid l N_{0}$ be a prime. For an element $q \in \mathbb{Q}$ whose denominator is not divisible by $p$, we denote by $\bar{q}$ the reduction of $q$ modulo $p$ in $\mathbb{F}_{p}$. If (1) has an integer solution $(x, y, z)$ with $p \mid x y z$ other than $(0,0,0)$, then necessarily

$$
\overline{a / b}, \overline{b / c} \text {, or } \overline{c / a} \in \mathbb{F}_{p}^{* n} \text {. }
$$

In this case we get from $\rho_{l}^{E_{n,(x, y)}} \simeq \rho_{l}^{f}$ that $a_{p}(f) \equiv \pm(p+1)(\bmod \lambda)$. If the (hypothetical) integer solution $(x, y, z)$ to (11) satisfies $p \nmid x y z$, then $(\bar{x}, \bar{y})$ belongs to

$$
S_{n, p}=\left\{(\alpha, \beta) \in \mathbb{F}_{p}^{*} \times \mathbb{F}_{p}^{*}: \bar{a} \alpha^{n}+\bar{b} \beta^{n}+\bar{c} \gamma^{n}=0 \text { for some } \gamma \in \mathbb{F}_{p}^{*}\right\} .
$$

For any $P=(\alpha, \beta) \in S_{n, p}$, define an elliptic curve over $\mathbb{F}_{p}$ by

$$
\mathcal{E}_{n, p, P}: y^{2}=x\left(x-\bar{a} \alpha^{n}\right)\left(x+\bar{b} \beta^{n}\right) .
$$

Then $a_{p}\left(E_{n,(x, y)}\right)$ belongs to

$$
\mathcal{A}_{n, p}=\left\{a_{p}\left(\mathcal{E}_{n, p, P}\right): P \in S_{n, p}\right\} .
$$

Also consider the set (of possibilities for $\operatorname{trace}\left(\rho_{l}^{E_{n}}\left(\operatorname{Frob}_{p}\right)\right)$ )

$$
\mathcal{T}_{n, p}=\left\{\begin{array}{l}
\mathcal{A}_{n, p} \cup\{ \pm(p+1)\} \quad \text { if (6) holds; } \\
\mathcal{A}_{n, p} \quad \text { otherwise. }
\end{array}\right.
$$

Hence, in order to prove that for a (hypothetical) solution $(x, y, z, n)$ to (11) and a certain newform class $f$ of level $N_{0}$ we cannot have $\rho_{l}^{E_{n,(x, y)}} \simeq \rho_{\lambda}^{f}$ for any prime 
$\lambda \subset \mathcal{O}_{f}$ lying above $l$, it suffices to find a prime $p \nmid N_{0} l$ such that

$$
l \nmid \prod_{a \in \mathcal{T}_{n, p}} \operatorname{Norm}\left(a-a_{p}(f)\right) .
$$

If for all newform classes $f$ of level $N_{0}$ we can find such a prime $p$, then we conclude that (11) has no integer solutions with $x y z \neq 0$. In practice, since we already computed $\mathcal{L}_{f, p_{\max }}$ for some 'large' prime $p_{\max }$, it only remains to find such a prime $p$ for the newform classes $f$ of level $N_{0}$ for which $l \in \mathcal{L}_{f, p_{\max }}$.

Remark 17. From a computational point of view, it is worthwhile to only consider $\mathcal{E}_{n, p, P}$ up to quadratic twist in order to determine $\mathcal{A}_{n, p}$ (and hence $\mathcal{T}_{n, p}$ ). To be specific, let

$$
S_{n, p}^{\prime}=\left\{\alpha \in \mathbb{F}_{p}^{*}: \overline{a / c} \alpha^{n}+\overline{b / c} \in \mathbb{F}_{p}^{* n}\right\}
$$

Then we get

$$
\mathcal{A}_{n, p}=\left\{ \pm a_{p}\left(\mathcal{E}_{n, p,(\alpha, 1)}\right): \alpha \in S_{k, p}^{\prime}\right\} .
$$

For every equation in Theorem 14, an entry $(l, p)$ under 'local $(l, p)$ ' of Table 1 indicates that $C_{l}\left(\mathbb{Q}_{p}\right)=\emptyset$. Furthermore, for every prime $l \geq 5$ and newform class $f$ of level $N_{0}$ for which $l \in \mathcal{L}_{f, p_{\max }}$ (which implies $l \in \mathcal{L}_{p_{\max }}$ ) and $C_{l}$ is locally solvable everywhere, there is an entry $(l, p)$ in Table 1 under 'Kraus $(l, p)$ ' indicating that $l \nmid \prod_{a \in \mathcal{T}_{l, p}} \operatorname{Norm}\left(a-a_{p}(f)\right)$. This completes the data that proves Theorem 14 for primes $l \geq 5$.

\begin{tabular}{c|c|c|c|c|c}
$(a, b, c)$ & level & $p_{\max }$ & $\mathcal{L}_{p_{\max }}-\{3\}$ & local $(l, p)$ & Kraus $(l, p)$ \\
\hline$\left(5^{2}, 2^{4}, 23^{4}\right)$ & 115 & 3 & $\{5\}$ & $(5,11)$ & - \\
$\left(5^{8}, 2^{4}, 37\right)$ & 185 & 3 & $\{5,19\}$ & $(19,19)$ & $(5,31)$ \\
$\left(5^{7}, 2^{4}, 59^{7}\right)$ & 295 & 3 & $\{5,7\}$ & $(5,5)$ & $(7,43)$ \\
$\left(7,2^{4}, 47^{7}\right)$ & 329 & 23 & $\{5\}$ & - & $(5,11)$ and $(5,41)$ \\
$\left(11,2^{4}, 5^{2} \cdot 17^{2}\right)$ & 935 & 71 & $\{5,7\}$ & $(5,5)$ & $(7,29)$ \\
\multicolumn{5}{c}{ TABLE 1. Data for primes $l \geq 5}$.
\end{tabular}

4.1.2. $n=9$. To prove that $C_{9}(\mathbb{Q})=\emptyset$ for our curves, we first note that $C_{9}$ has points everywhere locally; this is a straightforward computation. We can also quickly find a rational point on $C_{3}$ and find that the corresponding elliptic curve has positive rank over $\mathbb{Q}$; see Table 2 . Next, we start applying a mod-3 modular approach. Before we can apply level lowering, we need to show that $\rho_{3}^{E_{9}}$ is irreducible (which implies that it is absolutely irreducible). Later on, we shall need the stronger result that $\rho_{3}^{E_{9}}$ is strongly irreducible. Using Proposition 13 we obtain an explicit criterion for checking this.

Proposition 18. Suppose $p$ is a prime such that

- $p \nmid a b c$,

- $p \equiv 1(\bmod 9)$,

- condition (6) does not hold,

- for all $P \in S_{9, p}$ we have $3 \mid \# \mathcal{E}_{9, p, P}$.

Then $\rho_{3}^{E_{9}}$ is strongly irreducible. 
Notice that, as in Remark 17 by considering quadratic twists, the last condition in the proposition above can be replaced by: for all $\alpha \in S_{9, p}^{\prime}$ we have $3 \mid \# \mathcal{E}_{9, p,(\alpha, 1)}$. In all our examples, we find a prime $p=p_{\text {irr }}$ satisfying all the conditions in the proposition above, the value is recorded in Table 2 .

Although we would like to apply Theorem 2 with $r=2$ and $l=3$, for most of the levels $N_{0}$ we are considering there actually exist distinct pairs $\left(f_{1}, \lambda_{1}\right),\left(f_{2}, \lambda_{2}\right)$ of newform classes $f_{1}, f_{2}$ of level $N_{0}$ and prime ideals $\lambda_{i} \subset \mathcal{O}_{f_{i}}(i=1,2)$ lying above 3 for which $\rho_{f_{1}}^{\lambda_{1}} \simeq \rho_{f_{2}}^{\lambda_{2}}$ holds. So we start with applying 'ordinary' level lowering mod-3. For every newform class $f$ of level $N_{0}$ with $3 \in \mathcal{L}_{f, p_{\max }}$ we compute for various primes $p \nmid a b c$ with $p \equiv 1(\bmod 3)$ the set $\mathcal{T}_{9, p}$ and check if

$$
3 \nmid \prod_{a \in \mathcal{T}_{9, p}} \operatorname{Norm}\left(a-a_{p}(f)\right) .
$$

Denote by $\mathcal{N}_{p_{0}}$ the set of newform classes $f$ of level $N_{0}$ such that for all primes $p \leq p_{0}$, (7) does not hold. The prime $p_{0}$ we used, together with a description of $\mathcal{N}_{p_{0}}$ is given in Table2. Now if $\rho_{3}^{E_{9}} \simeq \rho_{\lambda}^{f}$ for some newform class $f$ of level $N_{0}$ and prime ideal $\lambda \subset \mathcal{O}_{f}$ lying above 3 , then necessarily $f \in \mathcal{N}_{p_{0}}$. For all the cases we consider, taking into account that the image of the representation has to be contained in $\mathrm{GL}_{2}\left(\mathbb{F}_{3}\right)$, we find that $\lambda$ has to be of inertia degree 1 . Furthermore, we find a $p_{0}$ such that $\mathcal{N}_{p_{0}}$ is small enough so that the uniqueness condition in Theorem 2 now holds. As for the ramification condition, in all our cases, except when $N_{0}=935$, we find that 3 is unramified in $K_{f}$ for all $f \in \mathcal{N}_{p_{0}}$. For the case where $N_{0}=935$, we actually find that the pair $(f, \lambda)$ we can not deal with modulo 3 has $\lambda$ ramified in $K_{f}$, and we deal with this case by studying the deformation ring directly. In all other cases, we apply Theorem 2 (with $r=2$ and $l=3$ ) to all newform classes in $\mathcal{N}_{p_{0}}$. This time, for all $f \in \mathcal{N}_{p_{0}}$, we simply try to find a prime $p \nmid 3 N_{0}$ such that for all $a \in \mathcal{T}_{p}$ we have

$$
9 \nmid \operatorname{Norm}\left(a-a_{p}(f)\right)
$$

and $\mathbb{Q}\left(a_{p}(f)\right)=K_{f}$. In all cases we readily do find such a prime $p$; for the value, see Table 2. This means that we also obtain a contradiction from $\rho_{9}^{E_{9}} \simeq \rho_{\lambda^{2}}^{f}$ for all $f \in \mathcal{N}_{p_{0}}$ and all relevant $\lambda$. It follows that $C_{9}(\mathbb{Q})=\emptyset$.

\begin{tabular}{c|c|c|c|c|c} 
level & $\mathbb{Q}$-rank of $C_{3}$ & $p_{\text {irr }}$ & $p_{0}$ & $\mathcal{N}_{p_{0}}$ description & $p$ \\
\hline 115 & 2 & 73 & - & $d=1$ & 2 \\
185 & 1 & 73 & 73 & $d=1^{*}$ & 2 \\
295 & 2 & 37 & 37 & $d=6$ & 13 \\
329 & 2 & 109 & 13 & $d=5, d=6$ & 5,5 \\
935 & 2 & 37 & 37 & $d=11^{*}$ & -
\end{tabular}

TABLE 2. Data for $n=3$ and $n=9 ; d$ denotes the degree of the newform class. In case of a $*$, the degree alone does not determine the class uniquely, in which case we impose the extra condition $\operatorname{trace}\left(a_{2}(f)\right)=0$, which does determine the class uniquely.

Remark 19. In other cases, using $\mathcal{T}_{p}$ might not give enough information. Using $\mathcal{T}_{9, p}$ instead might lead to the desired conclusion. Furthermore, computing $a_{p}(f)$ $(\bmod \lambda)$ may yield more information than $\operatorname{Norm}\left(a-a_{p}(f)\right)(\bmod l)$. For example, if 
$(a, b, c)=\left(7,2^{4}, 47^{7}\right)$, computing $\operatorname{Norm}\left(a-a_{p}(f)\right)(\bmod 3)$ did not give us enough information to rule out the newform classes of level 329 with degrees 5 and 6 . However, the newform class $f$ of degree 6 can be ruled out using $a_{p}(f)(\bmod \lambda)$. Specifically, there is a unique prime $\lambda$ above 3 of inertia degree 1 in $K_{f}$. For any other prime $\lambda^{\prime}$ above 3 , the image of the Galois representation $\rho_{\lambda^{\prime}}^{f}$ is not contained in $\mathrm{GL}_{2}\left(\mathbb{F}_{3}\right)$, hence not isomorphic to $\rho_{3}^{E_{9}}$. To rule out the prime $\lambda$ in this case, we just note that $f \bmod \lambda$ is an Eisenstein series, which means $\rho_{\lambda}^{f}$ is reducible, hence not isomorphic to $\rho_{3}^{E_{9}}$ since we proved this is irreducible.

In fact, using these observations, in all our cases we find that there is exactly one pair of $(f, \lambda)$ for which we are unable to obtain a contradiction using just level lowering modulo 3. In Section 5 we will prove that level lowering modulo 3 is not sufficient for this pair.

4.1.3. Level 935. We now consider the example

$$
11 x^{9}+2^{4} y^{9}+5^{2} \cdot 17^{2} z^{9}=0 .
$$

Using the data in Table 2, we conclude that if there is a nontrivial solution to this equation, then we have $\rho_{3}^{E_{9}} \nsucceq \rho_{\lambda}^{f_{11}}$, where $f_{11}$ is the newform class of level 935 with degree 11 and trace $\left(a_{2}\left(f_{11}\right)\right)=0$, and $\lambda \subset \mathcal{O}_{f_{11}}$ a prime lying above 3 of inertia degree 1. There are exactly two primes $\lambda_{1}, \lambda_{2} \subset \mathcal{O}_{f_{11}}$ lying above 3 of inertia degree 1. One is unramified, say $\lambda_{1}$, but the other, say $\lambda_{2}$ is ramified. As for the representations $\rho_{\lambda_{i}}^{f_{11}}$ for $i=1,2$, we quickly find that they are not isomorphic.

The case $\rho_{3}^{E_{9}} \simeq \rho_{\lambda_{1}}^{f_{11}}$ actually leads to a contradiction fairly easily by computing $\mathcal{T}_{9,31}=\{ \pm 8, \pm 32\}$ and $a_{31}\left(f_{11}\right) \equiv 0\left(\bmod \lambda_{1}\right)$.

The situation for $\left(f_{11}, \lambda_{2}\right)$ is a bit more delicate, specially since we can not apply our level lowering theorem in this situation. However we can still rule this case out by using the deformation ring directly. Assume that $\rho_{3}^{E_{9}} \simeq \rho_{\lambda_{2}}^{f_{11}}$. Then $\rho_{9}^{E_{9}}$ is a minimal deformation of $\rho_{\lambda_{2}}^{f_{11}}$. Therefore, this representation should correspond to a unique map $\mathbb{R}^{\text {univ }} \rightarrow \mathbb{Z} / 9 \mathbb{Z}$. However we can explicitly compute $\mathbb{R}^{\text {univ }}=\mathbb{T}$, as we did in remark 8 . Since $\left(f_{11}, \lambda_{2}\right)$ is not congruent to any other newform class of level 935 , we get that $\mathbb{T}=\left(\mathcal{O}_{f_{11}}\right)_{\lambda_{2}}$. Using SAGE or MAGMA we get that $\mathbb{Z}\left[a_{3}\left(f_{11}\right)\right] \subset \mathcal{O}_{f_{11}}$ with an index coprime to 3 . Therefore

$$
\mathcal{O}_{f_{11}} \otimes \mathbb{Z}_{3}=\mathbb{Z}_{3}[T] /<P(T)>
$$

where

$P(T)=T^{11}-T^{10}-25 T^{9}+26 T^{8}+222 T^{7}-225 T^{6}-827 T^{5}+705 T^{4}+1212 T^{3}-449 T^{2}-770 T-168$

is the minimal polynomial for $a_{3}\left(f_{11}\right)$. Factoring $P(T)$ over $\mathbb{Z}_{3}$, we can conclude that

$$
\mathbb{T}=\left(\mathcal{O}_{f_{11}}\right)_{\lambda_{2}}=\mathbb{Z}_{3}[T] /<T^{2}-a T+b>
$$

where $a \equiv 4(\bmod 9)$ and $b \equiv 7(\bmod 9)$. Looking at the above equation modulo 9 , we get $T^{2}-a T+b \equiv(x-2)^{2}+3(\bmod 9)$, which implies that there are no maps $\mathbb{T} \rightarrow \mathbb{Z} / 9 \mathbb{Z}$. This gives us a contradiction to our assumption that $\rho_{9}^{E_{9}}$ is a minimal deformation of $\rho_{\lambda_{2}}^{f_{11}}$, which rules this modular form out as well, and proves our result. 


\section{Necessity of LeVEl LOWERING MOdulo 9}

One can ask if we really needed level lowering modulo 9 for solving (11). A priori it could be possible that by using level lowering modulo 3 we can already obtain the desired contradictions. However, we will show that for our choice of Frey curve and triples $(a, b, c)$, level lowering modulo 3 does not yield enough information.

Remark 20. We note that there are Frey curves attached to the Diophantine equations

$$
\begin{aligned}
& a x^{n}+b y^{n}+c z^{3}=0 \\
& a x^{3}+b y^{3}+c z^{n}=0,
\end{aligned}
$$

and we can specialize these curves to the case $n=9$. The Frey curve attached to the first equation has a rational 3-isogeny by construction, so it is not suitable for level lowering. As for the Frey curves attached to the second equation, along similar lines as in Section 3, one can show that $E[3]$ is strongly irreducible. However, we end up having to deal with modular forms of higher level, for example when $(a, b, c)=\left(11,2^{4}, 5^{2} \cdot 17^{2}\right)$, we have to deal with modular forms of level (at least) 92565 , which is computationally very difficult.

We also note that other possible non-modular approaches to proving $C_{9}(\mathbb{Q})=\emptyset$ include descent methods and Mordell-Weil sieving. This is a promising approach, almost completely orthogonal to the modular method presented here and can be an interesting topic for further investigation.

Let $\overline{\mathcal{T}_{n, p}} \subset \mathbb{F}_{3}$ be the image of $\mathcal{T}_{3, p}$ under the reduction map modulo 3 . In this section we will show that for our examples, the unique pair of newform class $f$ of level $N_{0}$ and prime $\lambda \subset \mathcal{O}_{f}$ lying above 3 mentioned at the end of Remark 19. satisfies

$$
a_{p}(f) \quad \bmod \lambda \in \overline{\mathcal{T}_{9, p}}
$$

for all primes $p \nmid 3 N_{0}$. This means that level lowering modulo 9 (along with the argument in Section 4.1.3) is necessary to prove Theorem 14.

We will start by showing that $\overline{\mathcal{T}_{3, p}} \neq \mathbb{F}_{3}$ infinitely often, by showing $0 \notin \overline{\mathcal{T}_{3, p}}$ for infinitely many $p \equiv 1(\bmod 3)$.

Lemma 21. Let

$$
j: C_{n} \longrightarrow X(2) \longrightarrow X(1)
$$

be the $j$-invariant of the Frey elliptic curve corresponding to a point on $C_{n}$, let $p \equiv 1(\bmod n)$ be a prime with $p \nmid N_{0} n$, and let $\pi: X_{0}(3) \rightarrow X(1)$ be the natural forgetful map between the modular curves. Then

(1) $0 \notin \overline{\mathcal{T}_{n, p}}$ if and only if $j\left(C_{n}\left(\mathbb{F}_{p}\right)\right) \subset \pi\left(X_{0}(3)\left(\mathbb{F}_{p}\right)\right)$, if and only if

$$
\left(C_{n} \times_{X(1)} X_{0}(3)\right)\left(\mathbb{F}_{p}\right) \rightarrow C_{n}\left(\mathbb{F}_{p}\right)
$$

is surjective.

(2) $\pm 1 \in \overline{\mathcal{T}_{n, p}}$ if and only if there is a $z \in C_{n}\left(\mathbb{F}_{p}\right)$ such that $j(z) \in \pi\left(X_{0}(3)\left(\mathbb{F}_{p}\right)\right)$, if and only if $\left(C_{n} \times_{X(1)} X_{0}(3)\right)\left(\mathbb{F}_{p}\right)$ is not empty.

Proof. If $0 \notin \overline{\mathcal{T}_{n, p}}$, then by Lemma 12 we get that for all $z \in j\left(C_{n}\left(\mathbb{F}_{p}\right)\right)$ such that $z \neq \infty$ we have that the corresponding elliptic curve $\mathcal{E}_{n} / \mathbb{F}_{p}$ has a 3-isogeny, i.e. $z \in \pi\left(X_{0}(3)\left(\mathbb{F}_{p}\right)\right)$. We also know that $\infty \in \pi\left(X_{0}(3)\left(\mathbb{F}_{p}\right)\right)$, therefore we get $j\left(C_{n}\left(\mathbb{F}_{p}\right)\right) \subset \pi\left(X_{0}(3)\left(\mathbb{F}_{p}\right)\right)$. Now assume that $j\left(C_{n}\left(\mathbb{F}_{p}\right)\right) \subset \pi\left(X_{0}(3)\left(\mathbb{F}_{p}\right)\right)$. Let $z \in$ $j\left(C_{n}\left(\mathbb{F}_{p}\right)\right)$. Notice that if $z=\infty$ then the trace of Frobenius of the corresponding 
generalized elliptic curve is $\pm(p+1) \not \equiv 0(\bmod 3)$. If $z \neq \infty$, then by our assumption $z$ corresponds to an elliptic curve $\mathcal{E}_{n} / \mathbb{F}_{p}$ with a rational three isogeny, which by Lemma 12 will have trace of Frobenius equivalent to \pm 1 modulo 3. In either case we get $0 \notin \overline{\mathcal{T}_{n, p}}$. This proves the first equivalence. By definition of fiber products we see that $j\left(C_{n}\left(\mathbb{F}_{p}\right)\right) \subset \pi\left(X_{0}(3)\left(\mathbb{F}_{p}\right)\right)$ if and only if $\left(C_{n} \times_{X(1)} X_{0}(3)\right)\left(\mathbb{F}_{p}\right) \rightarrow C_{n}\left(\mathbb{F}_{p}\right)$ is surjective, which finishes the first part of the lemma.

The second part of the lemma is proved in a similar fashion.

Therefore, to find primes $p$ such that $0 \notin \overline{\mathcal{T}_{3, p}}$, we are reduced to finding $p$ such that $\left(C_{3} \times_{X(1)} X_{0}(3)\right)\left(\mathbb{F}_{p}\right) \rightarrow C_{3}\left(\mathbb{F}_{p}\right)$ is not surjective. For brevity, we will drop the $X(1)$ from the fiber product. Let $C_{3} \widetilde{\times X_{0}}(3)$ be the desingularization of the fiber product. The following lemma describes the map $C_{3} \widetilde{\times X_{0}}(3) \rightarrow C_{3}$.

Lemma 22. The curve $C_{3} \times X_{0}(3)$ is a genus one curve. Furthermore, the natural map $C_{3} \widehat{\times X_{0}}(3) \rightarrow C_{3}$ induces the multiplication by 2 on their Jacobians. In particular the Jacobian of $C_{3}$ is isomorphic to the Jacobian of $\widetilde{C_{3} \times X_{0}}(3)$.

Proof. A quick calculation shows that for every point $P$ of $X(1)$, the ramification indices of $X_{0}(3) \rightarrow X(1)$ above $P$, all divide the ramification indices of $C_{3} \rightarrow X(1)$ above $P$, which implies

$$
C_{3} \widetilde{\times X_{0}}(3) \longrightarrow C_{3}
$$

is unramified. Therefore, by Riemann-Hurwitz's theorem we get that $\widetilde{C_{3} \widetilde{\times X_{0}}}(3) \rightarrow$ $X(1)$ is a genus one curve.

To show that this map is the multiplication by the 2 map on the Jacobians, note that this a geometric statement, and it suffices to prove it for a particular twist of $C_{3}$. Specifically, we show that $C \widetilde{\times X_{0}}(3) \rightarrow C$ over $\mathbb{Q}$ induces the multiplication by 2 map on the Jacobians, where $C: x^{3}+y^{3}+z^{3}=0$. The induced map on the Jacobians

$$
\operatorname{Jac}(C) \longrightarrow \operatorname{Jac}\left(C \widetilde{\times X_{0}}(3)\right)
$$

is an isogeny of degree 4 , and is defined over $\mathbb{Q}$. Notice that $\operatorname{Jac}(C)$ is the elliptic curve given by $Y^{2}=X^{3}-2^{4} \cdot 3^{3}$. This elliptic curve has no rational 2 isogeny, therefore the only possibility is for the above map to be multiplication by 2 , which is the desired result.

The following proposition tells us that for most problems, $0 \notin \overline{\mathcal{T}_{3, p}}$ for infinitely many primes $p$.

Proposition 23. Let $J$ be the Jacobian of $C_{3} / \mathbb{Q}$ and let $p \equiv 1(\bmod 3)$ be a prime. Assume that $J$ has good reduction at $p$. Then $2 \mid a_{p}(J)$ if and only if $0 \in \overline{\mathcal{T}_{3, p}}$. Specifically if $4 a b c$ is not a perfect cube, then $0 \notin \overline{\mathcal{T}_{3, p}}$ infinitely often.

Proof. By Lemma21, we need to show that $2 \mid a_{p}(J)$ if and only if $\left(C_{3} \times X_{0}(3)\right)\left(\mathbb{F}_{p}\right) \rightarrow$ $C_{3}\left(\mathbb{F}_{p}\right)$ is not surjective. Notice that we can replace $C_{3} \times X_{0}(3)$ by its desingularization without loss of generality.

By Lemma 22, we know that $C_{3} \widetilde{\times X_{0}}(3)$ is a genus one curve. Using the Weil bound we get that genus one curves always have an $\mathbb{F}_{p}$ point, and hence they are isomorphic to their Jacobians. Let $P \in\left(C_{3} \widetilde{\times X_{0}}(3)\right)\left(\mathbb{F}_{p}\right)$, and let $Q$ be the 
image of this point in $C_{3}\left(\mathbb{F}_{p}\right)$. Using $P$ and $Q$ as the origins, we get an explicit isomorphism between $C_{3} \simeq J \simeq C_{3} \times X_{0}(3)$. Using this identification, the map $C_{3} \widetilde{\times X_{0}}(3) \rightarrow C_{3}$ is the multiplication by 2 map.

Now if we assume that $a_{p}(J)$ is odd, then $J\left(\mathbb{F}_{p}\right)$ is an Abelian group with an odd order, therefore the multiplication by 2 map is an isomorphism. In particular

$$
\left(C_{3} \widetilde{\times X_{0}}(3)\right)\left(\mathbb{F}_{p}\right) \rightarrow C_{3}\left(\mathbb{F}_{p}\right)
$$

is surjective. Similarly if $a_{p}(J)$ is even, then $J\left(\mathbb{F}_{p}\right)$ is an Abelian group with even order, and therefore multiplication by 2 map is not surjective on the $\mathbb{F}_{p}$ points.

Note that, when $4 a b c$ is not a perfect cube, the Jacobian $J$, given by (2), has no nontrivial rational 2-torsion point. In this situation, $a_{p}(J)$ is odd infinitely often, and we get that for infinitely many $p$ 's, $0 \notin \overline{\mathcal{T}_{3, p}}$. This completes the proof of the proposition.

In all our cases, it is easy to find an integer solution $(x, y, z)$ to (1) with $n=3$ such that $\rho_{3}^{E_{3,(x, y)}} \simeq \rho_{\lambda}^{f}$. This shows that $a_{p}(f) \bmod \lambda \in \overline{\mathcal{T}_{3, p}}$ for all primes $p \nmid 3 N_{0}$. However, since

$$
\overline{\mathcal{T}_{9, p}} \subset \overline{\mathcal{T}_{3, p}}
$$

a priori it is possible that Kraus' argument can succeed for some prime $p$ beyond our search space. This is in fact not the case, and we show that for $p$ large enough, this containment is in fact equality, and hence we can not get a contradiction (it is easy to see that for $p \equiv 2(\bmod 3)$ we have $\left.\overline{\mathcal{T}_{9, p}}=\overline{\mathcal{T}_{3, p}}=\mathbb{F}_{3}\right)$.

Proposition 24. (1) If $p>216^{2}$ is a prime congruent to 1 ( $\left.\bmod 3\right)$, then $\pm 1 \in \overline{\mathcal{T}_{3, p}}$ if and only if $\pm 1 \in \overline{\mathcal{T}_{9, p}}$.

(2) If $p>106^{2}$ is a prime congruent to $1(\bmod 3)$, then $0 \in \overline{\mathcal{T}_{3, p}}$ if and only if $0 \in \overline{\mathcal{T}_{9, p}}$.

Proof. To prove the first part of the claim, by Lemma 21, we need to show $C_{9} \times$ $X_{0}(3)$ has an $\mathbb{F}_{p}$ rational point, however this follows from the Weil bound

$$
\left|\left(C_{9} \widetilde{\times X_{0}}(3)\right)\left(\mathbb{F}_{p}\right)\right|>p+1-2 g \sqrt{p},
$$

where $g$ is the genus of $C_{9} \times X_{0}(3)$. To calculate this genus, note that $\widetilde{C_{9} \times X_{0}}(3) \rightarrow$ $C_{9}$ is unramified of degree 4 , and we know that the genus of $C_{9}$ is $(9-1)(9-2) / 2=$ 28. Therefore, using Riemann-Hurwitz's theorem we get that $g=109$. Therefore

$$
\left|\left(C_{9} \widetilde{\times X_{0}}(3)\right)\left(\mathbb{F}_{p}\right)\right|>p+1-218 \sqrt{p},
$$

which means for $p>218^{2}$, the curve $C_{9} \widetilde{\times X_{0}}(3)$ will have an $\mathbb{F}_{p}$ point. This finishes the proof of first part of the proposition.

To prove the second part, assume that $0 \in \overline{\mathcal{T}_{3, p}}$. Then, by Lemma 21 the map $\left(C_{3} \widehat{\times X_{0}}(3)\right)\left(\mathbb{F}_{p}\right) \rightarrow C_{3}\left(\mathbb{F}_{p}\right)$ is not surjective. Assume that $\left(\widetilde{C_{9} \times X_{0}}(3)\right)\left(\mathbb{F}_{p}\right) \rightarrow$ $C_{9}\left(\mathbb{F}_{p}\right)$ is surjective. We want to show that $p<106^{2}$. Let $P \in C_{9}\left(\mathbb{F}_{p}\right)$. Then we can find a point $Q \in\left(C_{9} \widetilde{\times X_{0}}(3)\right)\left(\mathbb{F}_{p}\right)$ which maps to $P$. This implies that $\phi(P)$ is in the image of $\left(\widetilde{C_{3} \times X_{0}}(3)\right)\left(\mathbb{F}_{p}\right) \rightarrow C_{3}\left(\mathbb{F}_{p}\right)$. However, since this map is just multiplication by 2 , and since it is not surjective, there are either 2 or 4 points that 


\begin{tabular}{c|c|c}
$(a, b, c)$ & $p_{0}$ & $p_{1}$ \\
\hline$\left(5^{2}, 2^{4}, 23^{4}\right)$ & $\{73,163\}$ & $\emptyset$ \\
$\left(5^{8}, 2^{4}, 37\right)$ & $\{73,307,541\}$ & $\{37\}$ \\
$\left(5^{7}, 2^{4}, 59^{7}\right)$ & $\{37,73,163,181,199,541\}$ & $\emptyset$ \\
$\left(7,2^{4}, 47^{7}\right)$ & $\emptyset$ & $\{109\}$ \\
$\left(11,2^{4}, 5^{2} \cdot 17^{2}\right)$ & $\{37,73,307,541\}$ & $\emptyset$
\end{tabular}

TABLE 3 . Primes $p$ where $\overline{\mathcal{T}_{3, p}} \neq \overline{\mathcal{T}_{9, p}}$.

map to $\phi(P)$. This implies that there are either 2 or 4 points in $\left(\widetilde{C_{9}} \widetilde{\times X_{0}}(3)\right)\left(\mathbb{F}_{p}\right)$ that map to $P$. Therefore

$$
\left|\left(C_{9} \widetilde{\times X_{0}}(3)\right)\left(\mathbb{F}_{p}\right)\right| \geq 2\left|C_{9}\left(\mathbb{F}_{p}\right)\right| .
$$

However, the Weil bound tell us that

$$
\begin{aligned}
\left|C_{9}\left(\mathbb{F}_{p}\right)\right| & \geq p+1-2 \cdot 28 \sqrt{p} \\
\left|\left(C_{9} \widetilde{X_{0}}(3)\right)\left(\mathbb{F}_{p}\right)\right| & \leq p+1+2 \cdot 109 \sqrt{p} .
\end{aligned}
$$

Using these estimates we get that $p<106^{2}$, as desired.

Remark 25. For the proof of Proposition 24, we used the most basic bounds for simplicity of the exposition. However, since the curves we are using are fairly special, much better bounds are known.

We can now readily find all primes $p$ such that $\overline{\mathcal{T}_{9, p}} \neq \overline{\mathcal{T}_{3, p}}$. Table 3 collects this data: the column labeled $p_{0}$ is the set of primes such that $0 \in \overline{\mathcal{T}_{3, p}}$ but $0 \notin \overline{\mathcal{T}_{9, p}}$, and the column labeled $p_{1}$ is the set of primes such that $\pm 1 \notin \overline{\mathcal{T}_{9, p}}$. For all such primes, we can check that $a_{p}(f) \in \overline{\mathcal{T}_{9, p}}$ which proves that we can not rule out $f$ using mod 3 level lowering.

\section{REFERENCES}

1. Henri Darmon, Fred Diamond, and Richard Taylor, Fermat's last theorem, Current developments in mathematics, 1995 (Cambridge, MA), Int. Press, Cambridge, MA, 1994, pp. 1-154.

2. Matt Darnell, Chris Holden, Ben Kane, Jared Weinstein, and Soroosh Yazdani, Msri modular forms summer workshop, http://www.math.ubc.ca/ syazdani/MSRI_archive, August 2006, Project proposed by William Stein.

3. Fred Diamond, On deformation rings and Hecke rings, Ann. of Math. (2) 144 (1996), no. 1, 137-166.

4. Luis Dieulefait and Xavier Taixés i Ventosa, Congruences between modular forms and lowering the level mod $l^{n}$, J. Théor. Nombres Bordeaux 21 (2009), no. 1, 109-118.

5. Emmanuel Halberstadt and Alain Kraus, Courbes de Fermat: résultats et problèmes, J. Reine Angew. Math. 548 (2002), 167-234.

6. Alain Kraus, Majorations effectives pour l'équation de Fermat généralisée, Canad. J. Math. 49 (1997), no. 6, 1139-1161.

7. $ـ$ Sur l'équation $a^{3}+b^{3}=c^{p}$, Experiment. Math. 7 (1998), no. 1, 1-13.

8. Kenneth A. Ribet, On modular representations of $\mathrm{Gal}(\overline{\mathbf{Q}} / \mathbf{Q})$ arising from modular forms, Invent. Math. 100 (1990), no. 2, 431-476.

9. Sympos. Pure Math., vol. 55, Amer. Math. Soc., Providence, RI, 1994, pp. 639-676.

10. Images of semistable Galois representations, Pacific J. Math. (1997), no. Special Issue, 277-297, Olga Taussky-Todd: in memoriam. 
11. Karl Rubin, Modularity of mod 5 representations, Modular forms and Fermat's last theorem (Boston, MA, 1995), Springer, New York, 1997, pp. 463-474.

12. Jean-Pierre Serre, Sur les représentations modulaires de degré 2 de $\mathrm{Gal}(\overline{\mathbf{Q}} / \mathbf{Q})$, Duke Math. J. 54 (1987), no. 1, 179-230.

13. Goro Shimura, Introduction to the arithmetic theory of automorphic functions, Publications of the Mathematical Society of Japan, vol. 11, Princeton University Press, Princeton, NJ, 1994.

14. Richard Taylor and Andrew Wiles, Ring-theoretic properties of certain Hecke algebras, Ann. of Math. (2) 141 (1995), no. 3, 553-572.

15. Andrew Wiles, Modular elliptic curves and Fermat's last theorem, Ann. of Math. (2) 141 (1995), no. 3, 443-551.

Department of Mathematics, The University of British Columbia, Room 121, 1984 Mathematics Road, Vancouver, B.C., Canada V6T 1Z2

E-mail address: dahmen@math.ubc.ca

Department of Mathematics \& Statistics, McMaster University, 1280 Main Street, West Hamilton, Ontario, Canada L8S 4K1

E-mail address: syazdani@math.mcmaster.ca 